Trauma Berufskrankh 2010 • 12[Suppl 2]:125-131 DOI 10.1007/s10039-010-1623-x

Online publiziert: 9 . Juni 2010

(c) Springer-Verlag 2010

\section{H. Zwipp}

Klinik und Poliklinik für Unfall- und Wiederherstellungschirurgie, Universitätsklinikum Carl Gustav Carus, Technische Universität Dresden

\title{
Arthrodese vs. Gelenkersatz
}

\section{Oberes Sprunggelenk}

\section{Prothese vs. Arthrodese - Pro und Kontra}

\section{Prothesenversorgung}

Trotz ermutigender Ergebnisse mit Sprunggelenkprothesen der dritten Generation belasten

- hohe Komplikations- und Revisionsraten bis $39 \%[1,7]$,

- Lockerungsraten bis 24\% [16] mit einer 5-Jahres-Überlebensrate von nur $70-89 \%[1,10]$ mit unsicheren Langzeitresultaten sowie

- zahlreiche Kontraindikationen, wie postinfektiöse Zustände, schlechte Weichteilverhältnisse, erhebliche Rückfußfehlstellung $[1,8,21,22]$,

die Indikation auch für diese neueren Prothesen. Eine noch unzulängliche Revisionsprothetik und teils große Defektstrecken nach notwendigem Ausbau auch der eher resektionsarmen 3-KomponentenProthesen erfordern dann eine sekundäre Arthrodese mit weitaus ungünstigeren Heilverläufen als bei primärer Arthrodese des oberen Sprunggelenks (OSG) [26].

\section{Arthrodese}

Die Arthrodese des oberen Sprunggelenkes stellt aus oben angeführten Gründen nach wie vor den Goldstandard mit einem gut definierten Risiko dar. Durch eine korrekt ausgeführte Arthrodese des oberen Sprunggelenkes im Sinne der Reorientierung aller Achsen sowie der Fusion in Rechtwinkelstellung (Neutralposition) des Fußes lassen sich zuverlässig bis zu $90 \%$ sehr gute und gute Langzeit- ergebnisse erreichen $[3,12,23]$. Nachteilig erscheinen kompensatorische Mehrbelastungen der nachgeschalteten Gelenke insbesondere des Subtalar- und ChopartGelenks mit der Entwicklung von so genannten Anschlussarthrosen. Derartige Problematiken werden aber am ehesten bei nicht rechtzeitig gestellter Indikation oder bei tibiotalarer Fusion in Fehlstellung gesehen $[5,12,25]$.

\section{Fußstellung}

Nach ganganalytischen Untersuchungen sowie Druckmessungen im Subtalargelenk in vitro sind Arthrodesen des oberen
Sprunggelenks in Spitzfußstellung biomechanisch ungünstiger als solche in Neutral-o-Stellung [2, 24]. Unter Beachtung von Druck- und Zugkraftvektoren im Fuß ist dem Zentrieren des Talus in der sagittalen und a.-p. Achse zur Tibia gegenüber der früher von der AO geforderten Rückversetzung der Vorzug zu geben $[3,14,23$, 25]. Letztere führt zu einer Verlängerung des Hebelarms des Rückfußes in Relation zur einwirkenden Kraft des M. triceps surae mit Aufbau unphysiologischer Druckverhältnisse im Subtalargelenk und Förderung einer so genannten Anschlussarthrose $[12,25]$.

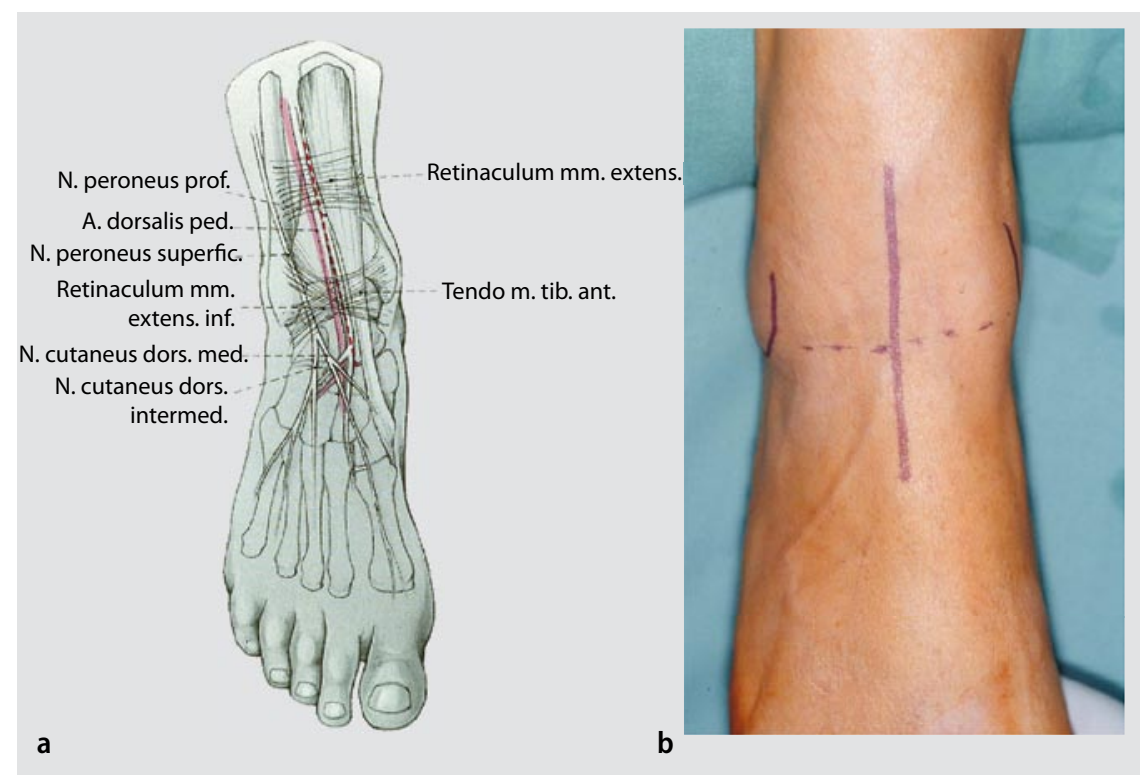

Abb. $1 \Delta$ Ventraler Zugang: 8-10 cm lange Längsinzision über der Mittellinie des vorderen, oberen Sprunggelenkes (a,b) lateral der Sehne des M. tibialis anterior. Nach Durchtrennung der Subkutis ist auf den extrafaszialen Verlauf der $\mathrm{Nn}$. cutaneus dorsalis intermedius et medialis zu achten, die den Operationssitus distal von lateral nach medial kreuzen können. Längsdurchtrennung der oberflächlichen Fascia cruris und z-förmige Spaltung des proximalen Extensorenretinaculums, um es später adaptieren zu können 

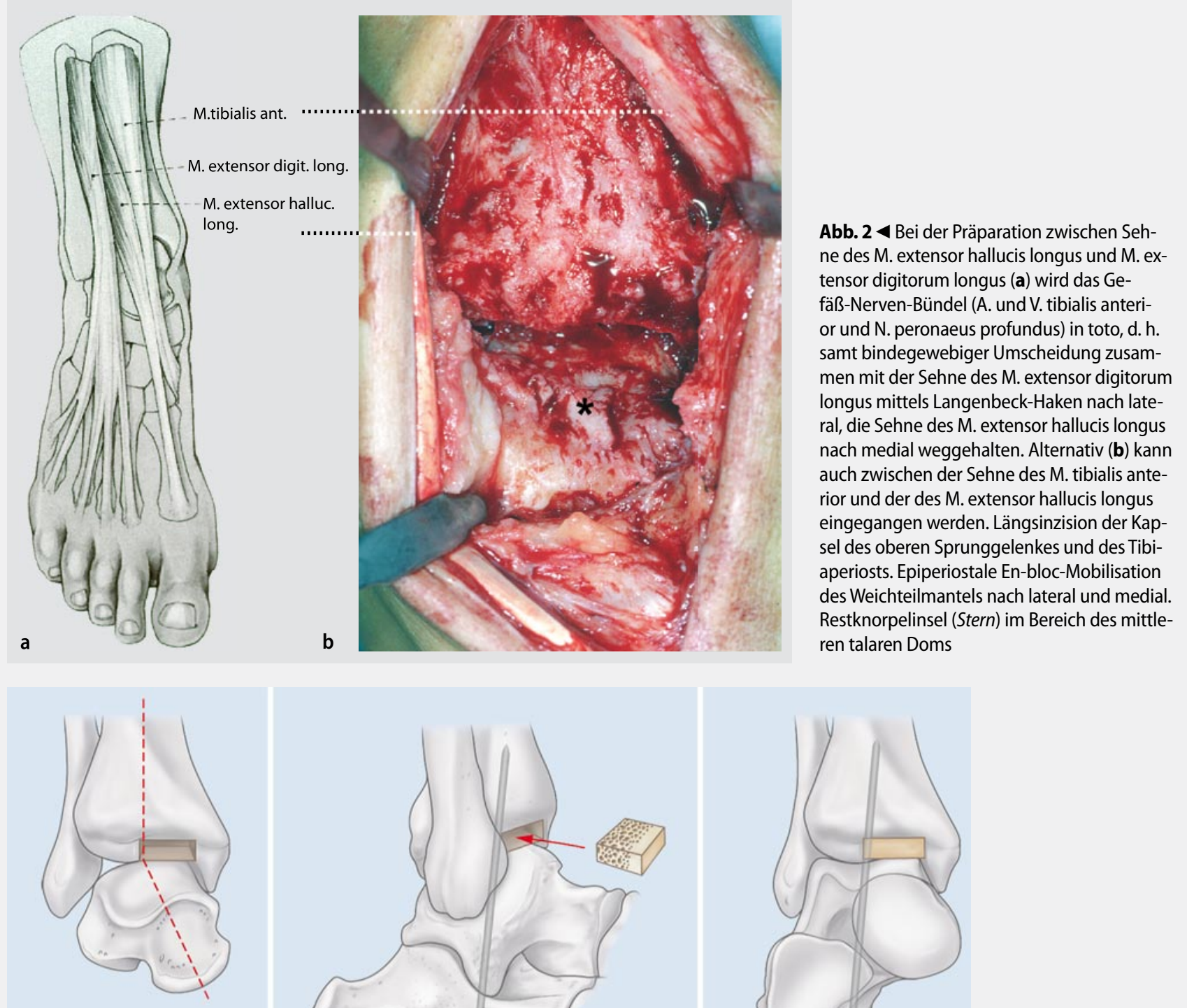

a
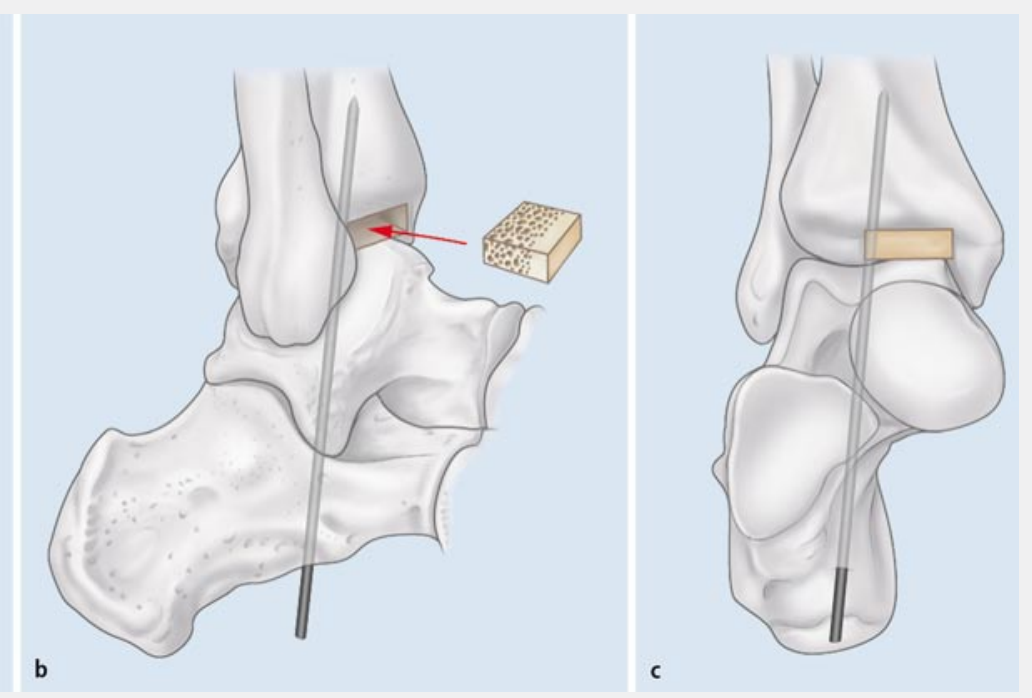

Abb. $3 \Delta$ Nach vollständiger Ausräumung des Restknorpels und der Sklerosezonen des medialen und lateralen Kompartiments muss jede Subluxationsstellung (a) des Talus im Sinne der Reorientierung durch exakte Reposition der Trochlea tali in die Knöchelgabel seitlich und a.-p. beseitigt $(\mathbf{b}, \mathbf{c})$ werden. Die bildwandlerkontrollierte Einstellung wird durch einen von plantar über den Kalkaneus temporär eingebrachten, 2,5 mm dicken Kirschner-Draht gesichert. In der Frontalebene werden Valgus- oder Varusfehlstellungen bei Resektion sklerosierter Areale durch Implantation eines kortikospongiösen Spans korrigiert (b), wobei dessen einzig verbleibende Kortex ventral unter Einbolzen positioniert wird

Ebenso ungünstig wirkt sich eine ventrale Translation des Talus mit Fuß gegenüber der Tibiaachse mit biomechanisch ungünstigen Hebelverhältnissen aus und behindert $\mathrm{u}$. U. die kompensatorische Überbeweglichkeit im nachgeschalteten Chopart-Gelenk.

Eine verbleibende Varusfehlstellung des Rückfußes führt zu einer Mehrbe- lastung der lateralen Fußsäule sowie des medialen Subtalargelenks, eine Valgusfehlstellung bewirkt eine Abflachung des Fußlängsgewölbes mit vermehrter Belastung des ersten Strahles verbunden mit einer relativen Fibulaverlängerung [12, 23]. 
Tab. 1 Anzahl und Art der Komplikationen ${ }^{\text {a }}$ bei 6 von 72 Patienten

\begin{tabular}{|c|c|c|}
\hline \multicolumn{2}{|c|}{ Komplikationen } & \multirow{2}{*}{$\begin{array}{l}\text { Anzahl } \\
\text { (n) } \\
2\end{array}$} \\
\hline Minor & $\begin{array}{l}\text { Oberflächliche } \\
\text { Weichteilinfektion }\end{array}$ & \\
\hline & Hämatomrevision & 3 \\
\hline & $\begin{array}{l}\text { Läsion des N. peronaeus } \\
\text { superficialis }\end{array}$ & 1 \\
\hline \multirow[t]{4}{*}{ Major } & Tiefe Weichteilinfektion & 0 \\
\hline & Osteitis & 0 \\
\hline & Ausbleibende Fusion & 1 \\
\hline & $\begin{array}{l}\text { Fehlstellungen } \\
\left(\text { Varus }>5^{\circ} \text { Nalgus }>10^{\circ} \text { ) }\right.\end{array}$ & 1 \\
\hline \multicolumn{3}{|c|}{$\begin{array}{l}\text { aschwerwiegende Komplikationen wie Osteitis, } \\
\text { tiefe Weichteilinfektion oder ausbleibende Fusi- } \\
\text { on traten nicht auf }\end{array}$} \\
\hline
\end{tabular}

die von 2 Schrauben, insbesondere wenn sie nicht gegenläufig positioniert sind [15]. Das eigene Vorgehen mit 4 Schrauben [25] zeigt klinisch ein Höchstmaß an Stabilität, sodass eine gipsfreie Nachbehandlung im Spezialstiefel möglich wird.

Die sparsame Resektion von Restknorpel und Sklerose unter knöchernem Erhalt der Sprunggelenkgabel führt im Gegensatz zur früher geübten Resektionsarthrodese zu einer höheren Stabilität und zur Vermeidung insbesondere von Rotationsfehlern $[12,14,25]$.

Im eigenen Verfahren erwies sich in den letzten 20 Jahren die standardisierte 4-Schrauben-Arthrodese als optimierte Konzeption, da sie nach Hansen [6] bei folgenden Prämissen eine spätere Rückführung zum Gelenkersatz erlaubt:

- Frühzeitige Indikation bei schmerzhafter Arthrose

- Vollständiger Erhalt der knöchernen Sprunggelenkgabel

- Physiologische Neutral-o-Stellung des Fußes unter Korrektur aller Achsen und zentraler Einstellung des Talus in Relation zur physiologischen Unterschenkelachse

- 4-Schrauben-Arthrodese in minimalinvasiver Technik

- Frühfunktionelle Nachbehandlung im flexiblen Arthrodesenstiefel mit Mobilisierung des Chopart-Gelenks

Indikationen zur reorientierenden 4-Schrauben-Arthrodese. Sie bestehen bei schmerzhafter, posttraumatischer oder idiopathischer Arthrose des oberen

Trauma Berufskrankh 2010 - 12[Suppl 2]:125-131 DOI 10.1007/s10039-010-1623-x

(c) Springer-Verlag 2010

\section{H. Zwipp \\ Arthrodese vs. Gelenkersatz. Oberes Sprunggelenk}

\section{Zusammenfassung}

Trotz ermutigender Ergebnisse mit Sprunggelenkprothesen der dritten Generation ist aufgrund deren hohen Komplikations-, Revisions- und Lockerungsraten sowie zahlreichen Kontraindikationen die Arthrodese des oberen Sprunggelenks nach wie vor als Goldstandard mit einem gut definierten Risiko anzusehen. Im eigenen Verfahren erwies sich in den letzten 20 Jahren die standardisierte 4-Schrauben-Arthrodese als optimierte Konzeption. Wird bei schmerzhafter Arthrose frühzeitig die Indikation zu einer Arthrodese des oberen Sprunggelenkes gestellt, wird operativ die knöcherne Sprunggelenkgabel erhalten, die Neutral-0-Stellung des Fußes unter zentraler Einstellung des Ta- lus zur physiologischen Unterschenkelachse in beiden Ebenen erzielt, kann in der minimalinvasiven Technik bei maximaler Stabilität der 4-Schrauben-Arthrodese eine frühfunktionelle Nachbehandlung im Variostabil ${ }^{-}$-Stiefel durchgeführt werden. Dadurch werden im Chopart-Gelenk zur Kompensation der Streckung und Beugung i.M. $24^{\circ}$, im AOFAS-Score (AOFAS: American Orthopaedic Foot and Ankle Society) auch im Langzeitergebnis über $90 \%$ sehr gute und gute Resultate erzielt.

\section{Schlüsselwörter}

Sprunggelenkprothese - Arthrose . 4-Schrauben-Arthrodese .

Oberes Sprunggelenk $\cdot$ Reorientierung

\section{Arthrodesis versus joint replacement. Upper ankle joint}

\section{Abstract}

Despite encouraging results with the third generation of ankle joint prostheses, the high complication, revision and loosening rates as well as many contraindications mean that arthrodesis of the ankle joint is still the gold standard with a well-defined risk factor. Our own modification of the standardized 4screw arthrodesis procedure has proven to be an optimized conception over the last 20 years. In cases of painful arthrosis, if the indications for arthrodesis of the upper ankle joint are recognized early enough, the bony convergence of the ankle joint can be operatively retained and the neutral position of the foot achieved by a central positioning of the talus relative to the anatomical axis of the lower leg axis in both planes. Using a minimally invasive technique by maximum stability of the 4-screw arthrodesis technique an early functional follow-up treatment can be carried out with the Vario-Stabil orthopedic boot. Using this method an average AOFAS score of 24 can be achieved for the transverse tarsal joint for compensation of stretching and bending and in the long-term over $90 \%$ very good and good results can also be achieved.

\section{Keywords}

Ankle joint prosthesis - Arthritis · Four-screw arthrodesis $\cdot$ Ankle joint $\cdot$ Reorientation 


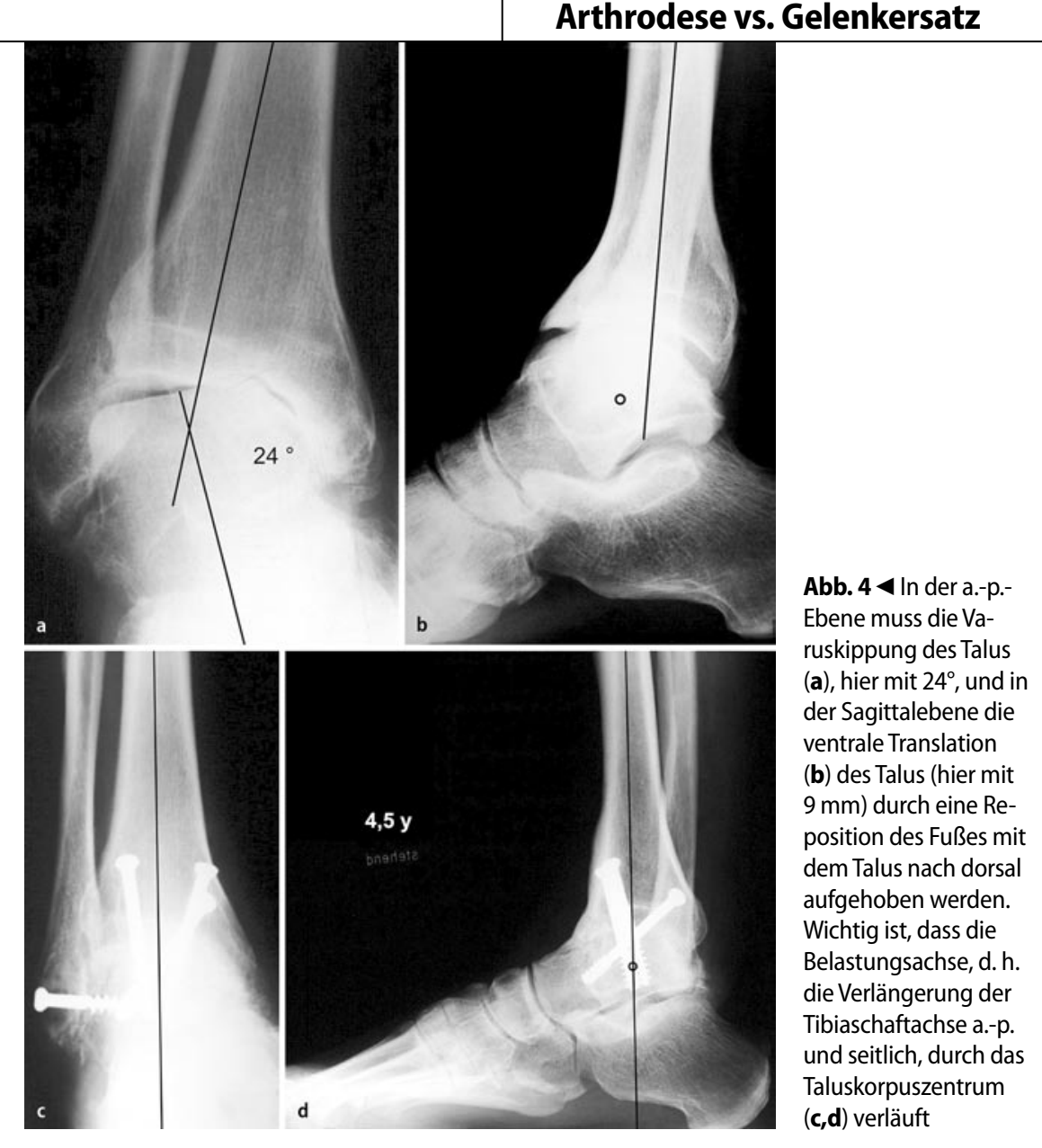

Sprunggelenkes, bei posttraumatischer Gelenkfehlstellung, die nicht Gelenk erhaltend zu rekonstruieren ist, bei Fußheberlähmung oder chronischer anterolateraler Rotationsinstabilität, die mit Gelenk erhaltenden Korrekturen wie Sehnentransfer oder Bandrekonstruktionen nicht zu beseitigen sind.

\section{Kontraindikationen zur reorientie-} renden 4-Schrauben-Arthrodese. Bei florider Osteitis, extremer Osteoporose, schlechten Haut- und Weichteilverhältnissen wie fortgeschrittener pAVK (periphere arterielle Verschlusskrankheit) oder dem Charcot-OSG ist eine 4-SchraubenArthrodese kontraindiziert.

\section{Präoperative Diagnostik}

Sie umfasst

- Belastungsaufnahmen beider oberer Sprunggelenke a.-p. in $20^{\circ}$ Innenrotation,

- Belastungsaufnahmen des Fußes mit oberem Sprunggelenk beidseits seitlich sowie

\section{Operationstechnik}

Bei liegender Blutsperre wird längs (- Abb. 1) mittig über dem oberen Sprunggelenk zugegangen, wobei das proximale Extensorenretinaculum z-förmig eröffnet wird, um eine Readaptation am Ende der Operation zu ermöglichen. Zwischen der Sehne des M. extensor hallucis longus und des M. tibialis anterior wird zum Sprunggelenk zugegangen, die Kapsel wird exzidiert, und Exophyten werden abgetragen ( $\bullet$ Abb. 2). Die Entfernung des Restknorpels und der Sklerosezonen wird mit feinen Meißeln, scharfen Löffeln, Lüer und Rangeur vorgenommen. Sobald ein mittelgroßer Arthrodesenspreizer eingesetzt werden kann, erfolgt schrittweise das vollständige Ausräumen des medialen und lateralen Kompartiments, wobei in den fibulotalaren Gelenkspalt oft nur kleine scharfe Löffel zur Entknorpelung eingeführt werden können. Wichtig ist, dass auch am Talushinterrand und an der Tibiahinterkante vollständig entknorpelt und die kontrakte Kapsel reseziert wird. Sollten Zweifel darin bestehen, ob die Sklerosezonen ausreichend abgetragen sind, muss die Blutsperre geöffnet und auf eine ausreichende Blutung aus dem Knochen geachtet werden. Ansonsten sind Pridie-Bohrungen und/oder eine zusätzliche Dekortikation notwendig. Sind Sklerosezonen so ausgedehnt, dass sie bei notwendiger Resektion zu einer Varus- oder Valgusstellung des Talus führen würden, ist eine Spongiosaoder monokortikale Transplantation vom gleichseitigen Beckenkamm notwendig (- Abb. 3). Zur Reposition des Talus in die exakte sagittale Längsachse der Tibia ist es oft erforderlich, mit voller Kraft den gesamten Fuß nach dorsal zu schieben, wobei die Tuchrolle als Hypomochlion zwingend notwendig ist. Sobald der Talus seitlich und a.-p. gut eingestellt ist, wird ein 2,5er Kirschner-Draht von der Planta pedis perkutan durch den Kalkaneus und den Taluskörper zentral in die Tibia eingebracht (• Abb.4). Unter kurzer Bildwandlerkontrolle wird in beiden Ebenen geprüft, ob das Taluszentrum in der Tibiaachse seitlich eingestellt ist und der Talus a.-p. in Bezug zur Tibia weder in einer Varus- noch in einer Valgusposition oder Translation steht. Sobald dies sicherge- 


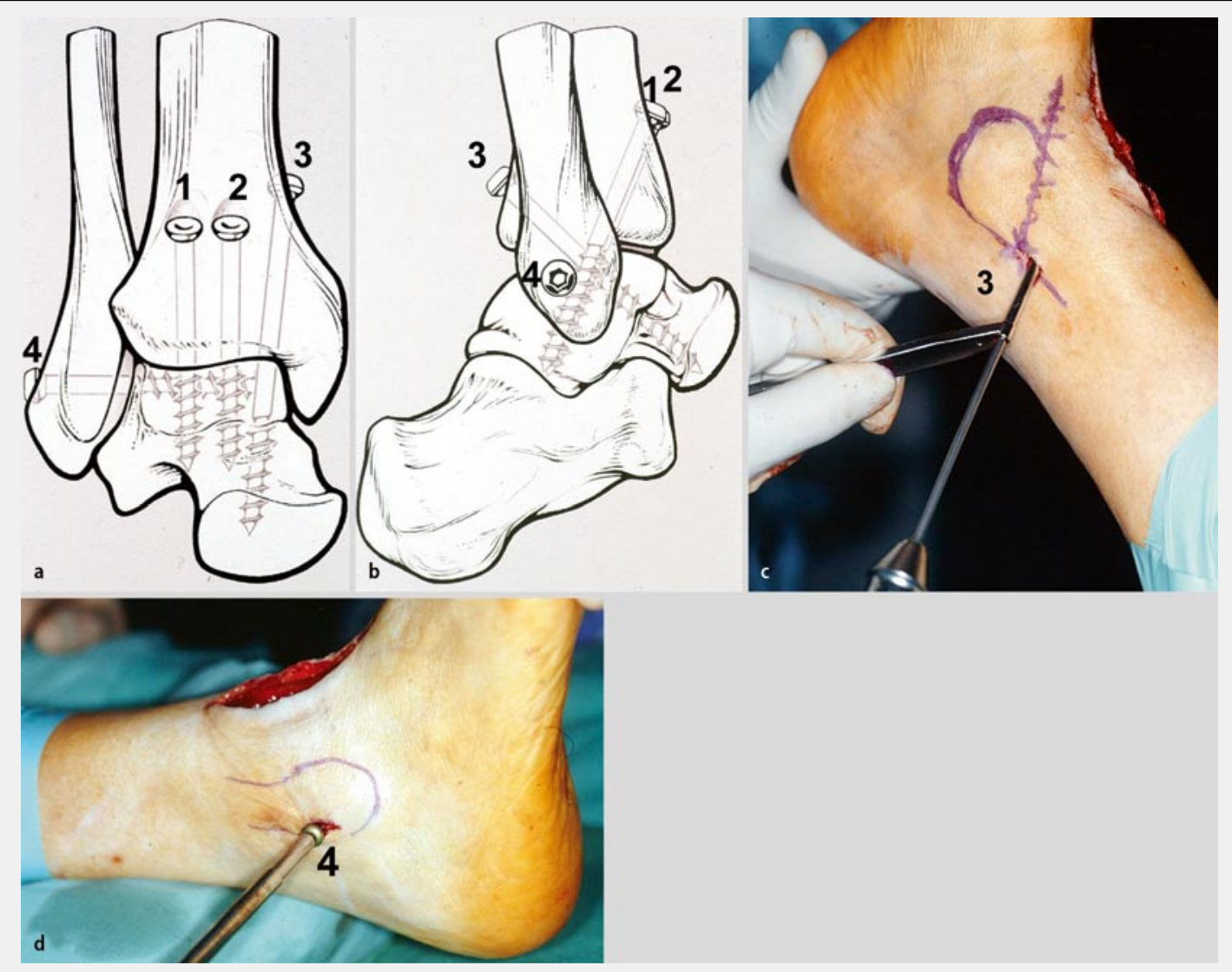

Abb. $5 \Delta$ Eine stabile Retention wird mit 4 6,5-mm-Spongiosazugschrauben (a,b) oder mit kanülierten, selbstschneidenden 7,3-mm-Spongiosazugschrauben erreicht. Die Fusion beginnt mit den ventralen Schrauben 1 und 2, welche etwa 3-4 cm oberhalb des Gelenkspaltes parallel zueinander und in einem Winkel von $10-20^{\circ}$ zum Tibiaschaft in die distale Tibia mit Zielrichtung in den medialen und lateralen Taluskörper platziert werden (ventraler Zuggurtungseffekt). Die 3. Schraube (a,b) wird über eine dorsomediale Stichinzision etwa 2,5 cm oberhalb des Innenknöchels (c) in den Talushals und in die ventrolaterale Portion des Taluskopfes eingebracht (dorsaler Zuggurtungseffekt). Die 4. Schraube $(\mathbf{a}, \mathbf{b})$ wird über eine Stichinzision (d) quer vom distalen Außenknöchel in den hinteren Taluskörper eingebracht, wodurch die Rotations- und transversale Stabilität erhöht werden. Zum festeren Gabelschluss ist bei Gabelerweiterung eine 5. Schraube supramalleolär empfehlenswert

stellt ist, kann mit der Verschraubung des oberen Sprunggelenkes begonnen werden. Das Setzen der ersten Schraube an der distalen Tibia beginnt lateral oder medial je nachdem, ob mit der 6,5er Spongiosazugschraube (16 mm Gewinde) noch eine geringe Valgisierung oder Varisierung des Taluskörpers erfolgen soll. Der Bohrer setzt etwa 3-4 cm oberhalb des Gelenkspalts tangential zur Tibiaschaftachse an und reicht in der Regel bei guter Platzierung über eine Strecke von $45-50 \mathrm{~mm}$ in den Taluskörper. Nach Setzen der ersten Schraube tibiotalar wird die zweite parallel hierzu gesetzt ( $\bullet$ Abb. 5). Während diese Schrauben über den offenen Situs eingebracht werden, wird die 3. Schraube über eine perkutane Inzision etwa 2,5 cm oberhalb des Innenknöchels am Hinterrand der Tibiaumschlagskante gesetzt. Nach Inzision wird mit dem kleinen Raspato- rium das Weichgewebe abgeschoben und überprüft, dass auf keinen Fall ein Konflikt mit der M.-tibialis-posterior-Sehne beim Bohren entsteht, weshalb die Gewebeschutzhülse bis an den Knochen herangeführt werden muss. Über diese Inzision wird beim durch den Assistenten hochgehobenen Unterschenkel in Richtung laterale Taluskopfportion gezielt, wobei diese Schraube durchaus eine Länge von 60$65 \mathrm{~mm}$ haben kann und immer die Verwendung eines 32-mm-Gewindes ermöglicht, wohingegen bei tibiotalaren Schrauben meist nur ein 16-mm-Gewinde möglich ist. Bei schlechter Knochenqualität sind Schrauben mit durchgehendem Gewinde zu empfehlen. Nach Setzen der 3. Schraube ist die wesentliche Stabilität im oberen Sprunggelenk hergestellt. Zum Heranpressen der Fibula an die laterale Wange des Talus wird eine 4. Schraube ebenfalls über Stichinzision mittig über der distalen Fibula in die hintere Portion des Taluskörpers eingebracht. Auch diese erlaubt in der Regel die Verwendung eines 32-mm-Gewindes. Nur bei vorbestehender Knöchelgabelinstabilität und notwendiger Ausräumung der Incisura fibularis tibiae käme eine 5. Schraube zur Erhöhung der Knöchelgabelinstabilität 2-3 cm oberhalb des Gelenkspalts ebenfalls über Stichinzision in Betracht. Sollte nach Setzen der ersten beiden Schrauben ein Konflikt mit dem eingebrachten 2,5$\mathrm{mm}$-Kirschner-Draht bestehen, kann dieser entfernt werden, ansonsten nach Setzen der 4. Schraube. Zum Abschluss der Operation erfolgt die Kontrolle der Achsenverhältnisse mit 2 Röntgenstandardprojektionen des oberen Sprunggelenks a.-p. und seitlich. 


\section{Arthrodese vs. Gelenkersatz}
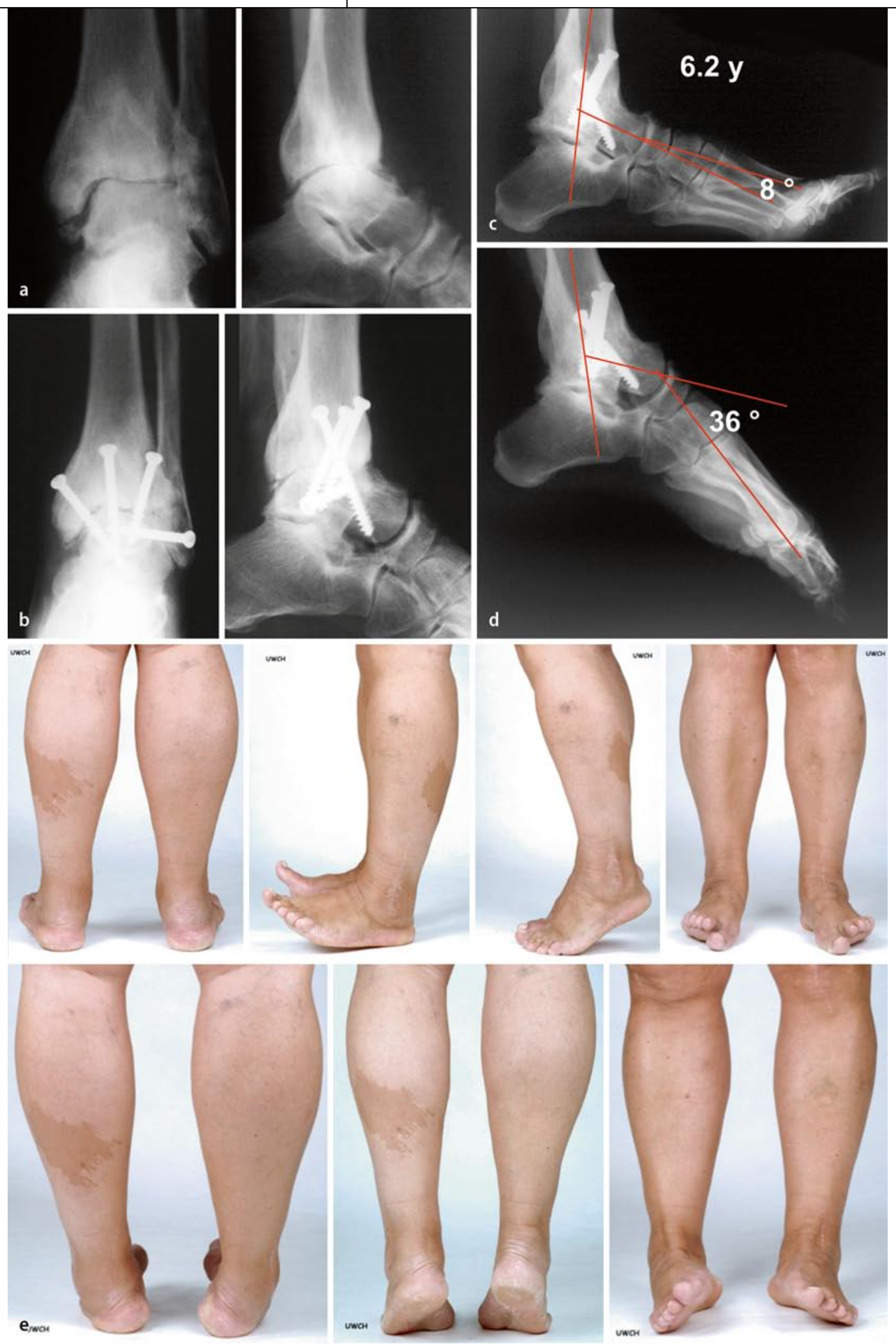

Abb. 6 Fallbeispiel einer 44-jährigen Patientin, 2 Jahre nach operativ versorgter SE-Fraktur (Supinations-Eversions-Fraktur) Stadium 4 und bereits weit fortgeschrittener Arthrose (Bargon 3) mit Sklerose der gelenknahen Tibia und des Taluskörpers (a). Postoperative 4-Schrauben-Arthrodese (b). Nach 6,2 Jahren hat die Patientin aufgrund frühfunktioneller postoperativer Behandlung im Variostabil $^{\oplus}$-Stiefel einen radiologischen Gesamtumfang der Bewegung im ChopartGelenk von $44^{\circ}(\mathbf{c}, \mathbf{d})$. Die Gesamtfunktion für Streckung, Beugung, Pro- und Supination ist sehr gut (e). Die Patientin ist schmerzfrei, voll arbeitsfähig und weist einen AOFAS-Punktewert von 92 aus

Nach Lösen der Blutsperre erfolgen eine subtile Blutstillung, Spülung und das Einlegen einer Redon-Drainage (10 Charr). Das proximale Extensorenretinaculum wird mit Einzelknopfnähten versorgt. Nach Subkutan- und Hautnaht werden ein steriler Wattekompressionsverband und ein Unterschenkelspaltgips angelegt, mit Lagerung auf einer Volkmann-Schiene.

\section{Nachbehandlung}

Der im Operationssaal angelegte Unterschenkelspaltgipsverband verbleibt zur Spitzfußprophylaxe und zum aktiven 
Leerpumpen der plantaren Fußsohlenplexus mit aktivem Drücken des Fußes gegen die Gipssohle bis zur Wundheilung.

Ab dem 4.-6. postoperativen Tag wird der so genannte flexible Arthrodesenstiefel (Variostabil ${ }^{-}$-Schuh, Fa. Busch GmbH, - Infobox 1) angelegt, und es werden Gehübungen einschließlich Treppensteigen physiotherapeutisch initiiert. Die volle Belastung im Arthrodesenstiefel ist bei Schmerzfreiheit erlaubt. Nur bei notwendiger Spongiosatransplantation sollte eine Teilbelastung mit 196,2 N (20 kp) für 612 Wochen empfohlen werden. Am dem Zeitpunkt der vollen Belastung und Bewegung des Fußes im Arthrodesenstiefel (etwa $20^{\circ}$ ) ist eine niedermolekulare Heparinbehandlung nicht mehr zwingend notwendig. Klinisch-radiologische Kontrollen werden nach 6 Wochen, abschließend nach 12 Wochen empfohlen.

\section{Ergebnisse}

Es konnten 72 Patienten mit einem Durchschnittsalter von 53 Jahren (34-69 Jahre) nach durchschnittlich 5,9 Jahren (4,87,8 Jahre) klinisch und röntgenologisch nachuntersucht und nach den AOFASKriterien (AOFAS: American Orthopaedic Foot and Ankle Society) [9] bewertet werden (• Abb. 6). Der mittlere AOFASScore von präoperativ 36 erhöhte sich auf 85 Punkte.

Komplikationen wie tiefe Weichteilinfektion, Osteitis oder ausbleibende Fusionen sind in $\square$ Tab. 1 zusammengefasst. Lediglich bei 1 Patienten mit hereditärer motorisch-sensorischer Neuropathie (Morbus Charcot-Marie-Tooth) kam es zur Pseudarthrose und zu einem Korrekturverlust von $15^{\circ}$ im Sinne der Varusfehlstellung.

Zum Zeitpunkt der Nachuntersuchung hatte eine zuvor schon bestehende Anschlussarthrose im Subtalargelenk bei 13 von 43 Patienten und im Talonavikulargelenk bei 5 von 26 Patienten zugenommen. Bei den meisten Patienten blieben die Anschlussarthrosen unverändert.

Im Chopart-Gelenk konnte anhand von radiologischen Funktionsaufnahmen eine durchschnittliche Beweglichkeit von $24^{\circ}\left( \pm 18^{\circ}\right)$ festgestellt werden.

\section{Besonderheiten}

Bei unzureichendem Schraubenhalt sollten in jedem Fall Schrauben mit durchgehendem Gewinde verwendet werden.

Sollte sich der Talus nicht ausreichend weit genug nach dorsal in der Sprunggelenkgabel einstellen lassen, sodass sein Zentrum nicht bis in die Achse der Tibia reicht, ist in jedem Fall nochmals zu überprüfen, ob nicht dorsale Kapselreste eine ausreichende Reposition nach dorsal verhindern.

Lässt sich der Talus nicht aus einer Equino-varus-Fehlstellung korrekt einstellen, kann zusätzlich eine perkutane Achillessehnentenotomie notwendig werden.

Die notwendige Resektion sklerotischer Zonen im Tibiaplafond oder im Talus kann eine Überlänge der Fibula zur Folge haben. Diese muss zur Vermeidung eines Impingements verkürzt werden, sofern nicht durch eine Spongiosa- oder kortikospongiöse Transplantation ein Längenausgleich erzielt werden kann.

\section{Infobox 1 Bezugsadresse}

- Fa. Busch GmbH, Lemlinstraße 9, 74080 Heilbronn

\section{Korrespondenzadresse}

\section{Prof. Dr. H. Zwipp}

Klinik und Poliklinik für Unfallund Wiederherstellungschirurgie, Universitätsklinikum Carl Gustav Carus, Technische Universität Dresden, Fetscherstraße 74, 01307 Dresden Hans.Zwipp@uniklinikum-dresden.de

Interessenkonflikt. Keine Angaben

\section{Literatur}

1. Anderson T, Montgomery F, Carlsson A (2003) Uncemented STAR total ankle prostheses. J Bone Joint Surg Am 85:1321-1329

2. Buck P, Morrey BF, Chao EY (1987) The optimum position of arthrodesis of the ankle. J Bone Joint Surg Am 69:1052-1062

3. Endres T, Grass R, Rammelt S, Zwipp H (2005) Die Vier-Schrauben-Arthrodese des oberen Sprunggelenks. Oper Orthop Traumatol 17:345-360

4. Friedmann RL, Glisson RR, Nunley JA 2nd (1994) A biomechanical analysis of two techniques for tibio-talar arthrodesis. Foot Ankle Int 15:301-305

5. Grass R, Zwipp H (1998) Die Arthrodese des oberen Sprunggelenkes in der Vier-Schrauben-Technik. Oper Orthop Traumatol 10:134-142
6. Hansen ST (2005) Grundsätzliche Überlegungen zur Technik der Sprunggelenkarthrodese im Hinblick auf einen späteren Wechsel zur Sprunggelenkendoprothese. Bemerkungen eines eingeladenen Kommentators. Oper Operat Traumatol 17:563568

7. Hintermann B, Valderrabano V (2001) Endoprothetik am oberen Sprungelenk. Z Ärztl Fortbild Qualitätssich 95:187-194

8. Hintermann B, Valderrabano V (2003) Total ankle replacement. Foot Ankle Clin N Am 8:375-405

9. Kitaoka HB, Alexander IJ, Adelaar RS (1994) Clinical rating systems for the ankle-hindfoot, midfoot, hallux and lesser toes. Ankle Int 15:349-352

10. Knecht SI, Estin M, Callagan JJ et al (2004) The agility total ankle arthroplasty: seven to sixteen year follow-up. J Bone Joint Am 86:1161-1171

11. Kofoed $H$ (1995) Cylindrical cemented ankle arthroplasty: a prospective series with long-term follow-up. Acta Orthop Scand Suppl 231:8

12. Morgan CD, Henke JA, Bailey RW, Kaufer H (1985) Long-term results of tibiotalar arthrodesis. J Bone Joint Surg Am 67:546-550

13. Morrey BF, Wiedemann GP (1980) Complications and long-term results of ankle arthrodesis following trauma. J Bone Joint Surg Am 62:777-784

14. Müller EJ, Wick M, Muhr G (1999) Chirurgische Therapie bei Inkongruenzen und Arthrosen am oberen Sprunggelenk. Orthopädie 28:529-537

15. Ogilvie-Harris DJ, Fitsialos D, Hedmann TP (1994) Arthrodesis of the ankle. A comparison of two versus three screw fixation in a crossed configuration. Clin Orthop 304:195-199

16. Pyevich MT, Saltzman CL, Callaghan JJ, Alvine FG (1998) Total ankle arthroplasty: a unique design. Two to twelve-year follow-up. J Bone Joint Surg Am 80:1410-1420

17. Saltzman CL, El-Khoury GY (1995) The hindfoot alignment view. Foot Ankle Int 16:572-576

18. Schill S, Biehl C, Thabe H (1998) Orthopädische Versorgung des Sprunggelenks: mittelfristige Ergebnisse nach Thompson-Richards- und STAR-Prothesen. Orthopäde 27:183-187

19. Scranton PE, Fu FH, Brown TD (1980) Ankle arthrodesis: a comparative clinical and biomechanical evaluation. Clin Orthop 151:234-243

20. Siebel T, Käfer W (1998) Behandlungsalternativen der schweren OSG-Arthrodese - Arthrodese versus Totalendoprothese. Med Welt 49:146-156

21. Strohecker T, Dávid A (2002) Die Endoprothetik des oberen Sprunggelenkes - eine echte therapeutische Alternative bei posttraumatischer Arthrose? Unfallchirurg 105:619-623

22. Thermann H, Saltzman CL (2002) Endoprothetischer Ersatz des oberen Sprunggelenkes. Unfallchirurg 105:496-510

23. Thermann H, Hüfner T, Roehler A et al (1996) Schraubenarthrodese des oberen Sprunggelenkes: Technik und Ergebnisse. Orthopäde 25:166-176

24. Thordarson DB, Markolf K, Cracchiolo A (1992) Stability of an ankle arthrodesis fixed by cancellousbone screws compared with that fixed by an external fixator. J Bone Joint Surg Am 74:1050-1055

25. Zwipp H (1994) Chirurgie des Fußes. Springer, Berlin Heidelberg New York, S 188-220

26. Zwipp H, Grass R (2005) Arthrodesen des oberen Sprunggelenks nach Endoprothesenfehlschlägen. Oper Orthop Traumatol 17:518-533 